this cohort were already on optimal HF treatment, many being asymptomatic and had low NT-proBNP levels. Some patients were also ineligible for SGLT-2i because of Stage 4 CKD.

One-third of the diabetic patients in this HFrEF cohort were not at target $\mathrm{HbA1C}$ range and according to the ADAEASD Guidelines, all these patients should have SGLT-2i added to intensify glycaemic control. Lately, the Canadian Heart Society have updated their guidelines with a strong recommendation to introduce SGLT-2i in diabetics with ischemic cardiomyopathy despite adequate glycaemic control for cardiovascular benefits.

SGLT-2i represents an important, but underutilized therapeutic option by cardiologists, likely due to the lack of familiarity on its use. This study reveals that SGLT-2i prescription could potentially increase in HFrEF patients with or without T2DM as guidelines will soon be updated based on robust evidence from large-scale clinical trials and when prescribers become aware of the indication for primary prevention of heart failure hospitalization and death.

\section{THE USEFULNESS OF THE EXISTING GUIDELINES FOR PERFORMANCE OF ENDOMYOCARDIAL BIOPSY IN PATIENTS WITH SUSPECTED MYOCARDITIS AND RELATED OUTCOMES IN A CONTEMPORARY ERA}

${ }^{1} \mathrm{~L}$ Murphy, ${ }^{1} \mathrm{M}$ McGuckin, ${ }^{1} \mathrm{G}$ Giblin, ${ }^{2} \mathrm{~A}$ Keogh, ${ }^{2} \mathrm{~B}$ McGovern, ${ }^{2} \mathrm{~A}$ Fabre, ${ }^{1} \mathrm{~J} \mathrm{O}^{\prime} \mathrm{N}$ eill, ${ }^{1} \mathrm{~N}$ Mahon, 'E Joyce. 'Mater Misericordiae University Hospital, Dublin, Ireland; ' ${ }^{1}$ St Vincent's University Hospital, Elm Park, Dublin 4, Ireland

\subsection{6/heartjnl-2020-ICS.25}

Introduction Diagnostic endomyocardial biopsy (EMB) in patients with myocarditis helps to direct therapy and guide prognosis. The original 2007 joint scientific statement provided guideline indications based on unique clinical scenarios, detailing who should undergo this invasive investigation but have not been studied in a contemporary cohort of suspected myocarditis patients.

Purpose To investigate the correlation between the clinical guideline indications for EMB and the presence of a diagnostic biopsy result and associated outcomes in patients with suspected myocarditis in a national quaternary referral center.

Methods All cases of suspected myocarditis referred to the National Advanced Heart Failure and Transplant Center between 2009-2019 were identified through pathological records. A thorough retrospective chart review was then performed on all patients. Outcomes including need for inotrope or mechanical circulatory support (MCS), heart transplantation and in-hospital mortality were recorded.

Results In total, 25 (68\% male, mean age of $45 \pm 15$ years) EMBs were performed for suspected myocarditis between 2009-2019, 64\% $(n=16)$ of which demonstrated diagnostic results. Clinical characteristics of those with histologically confirmed myocarditis are represented in figure 1. Regarding pathologic subtypes, $81 \%(\mathrm{n}=13)$ identified an acute lymphocytic myocarditis, $13 \%(n=2)$ giant cell myocarditis and one patient $(6.3 \%)$ eosinophillic myocarditis. The majority of those with a histologically confirmed myocarditis had a Class I or IIa guideline indication for $\operatorname{EMB}(n=12,75 \%)$. All patients requiring inotropes and/or MCS $(n=9)$ and/or heart transplant $(n=3)$ were in this group. The remaining 4 patients $(25 \%)$, of whom three were diagnosed with acute lymphocytic

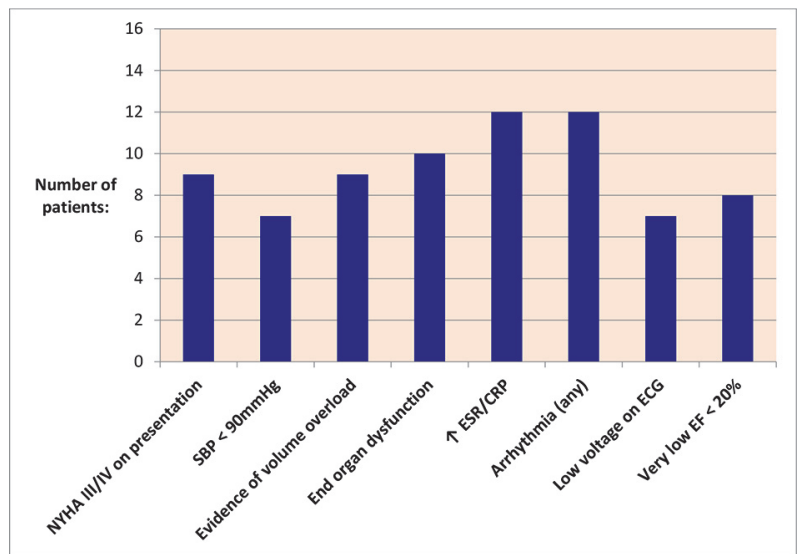

Abstract 25 Figure 1 Clinical characteristics of those with histologically confirmed myocarditis Abbreviations: CRP: C-reactive protein; ECG: electrocardiogram; EF: ejection fraction; ESR: erythrocyte sedimentation rate; NYHA: New York Heart Association; SBP: systolic blood pressure

myocarditis and one an immune checkpoint inhibitor (ICI) myocarditis, either met Class IIb criteria $(n=2)$ or would not have been accounted for in this guideline. Four patients $(25 \%)$ died during the index admission, one of whom was in the latter group (histologically confirmed myocarditis without a 2007 guideline indication).

Conclusions In this National referral sample, $75 \%$ of patients with suspected myocarditis had a Class I or IIa indication for $\mathrm{EMB}$, reinforcing the usefulness of these guidelines even in a contemporary era. Further, existing guideline indications appeared to identify a sicker group of patients more frequently requiring inotropes, MCS and/or heart transplant. However, in the contemporary era, $25 \%$ of patients had either none or a less well established indication for EMB despite a subsequent confirmed histological diagnosis, including a case of immune checkpoint inhibitor myocarditis, which has emerged since the publication of the 2007 guidelines. This highlights the need for clinical suspicion and correlation outside of accepted clinical scenarios.

\section{TEMPORAL CHANGE IN THE USE OF SODIUM GLUCOSE CO-TRANSPORTER 2 INHIBITORS}

G Offiah, C O'Connor, B Khan, D Moore. Tallaght University Hospital, Dublin, Ireland

\subsection{6/heartjnl-2020-ICS.26}

Introduction Diabetes mellitus (DM) is a major risk factor for cardiovascular disease (CVD). The EMPA-Reg study showed sodium glucose co-transporter 2 (SGLT2) inhibitors reduce the risk of death by $38 \%$ compared to placebo in diabetic patients with established CVD. These drugs have emerged as a major treatment option to reduce cardiovascular mortality as well as hospitalisation due to heart failure. The recent European Society of Cardiology (ESC) guidelines for DM has highlighted as a class I recommendation, the use of SGLT2 inhibitors as first line therapy in diabetics with established CVD or at high risk of CVD. This audit aims to determine whether the prescribing practices of SGLT2 inhibitors was influenced by the emergence of cardiovascular prevention data amongst patients with type 2 DM. 
Methods Retrospective cohort analysis was performed on all patients admitted to the coronary care unit (CCU) between January 2011 and September 2019. A review of the electronic health records of patients with new or established type 2 DM, who had been admitted with acute coronary syndrome (ACS) or heart failure (HF) as their primary diagnosis code was performed. Discharge prescriptions were analysed to determine which patients had been prescribed a SGLT2 inhibitor. The admission creatinine and estimated glomerular filtration rate (eGFR) for all patients were recorded. eGFR $\geq 60$ was used as the eligibility threshold for initiation of treatment with SGLT2 inhibitor as per summary of product characteristics. This data was analysed to determine whether there was a temporal change in prescriptions of this drug class. Statistical analysis was performed using standard Bayesain statistics.

Results There were 6870 patients admitted to the CCU between January 2011 and September 2019. 1054 patients had a diagnosis of type $2 \mathrm{DM}$ and were admitted with ACS or HF. 77 patients were excluded from the study due to incomplete data. Thus, 977 patients were included in the final data set for analysis. There were 54 newly diagnosed diabetics and 923 with established type 2 DM. 865 patients were admitted with ACS and 112 patients with HF. The ratio of male to female was 2.6:1. The mean age of the patient cohort was 65 .

There was a total of 40 patients prescribed SGLT2 inhibitors. Prior to the EMPA-Reg study in 2015, there were 4 of 387 eligible patients prescribed SGLT2 inhibitors, compared to 36 of 221 eligible patients thereafter. Chi-square statistic 45.1429 ( $\mathrm{p}<0.00001$ ). Temporal analysis from 2015 to 2019 showed increase in use from $5.5 \%$ to $20.5 \%$ (figure 1 ).

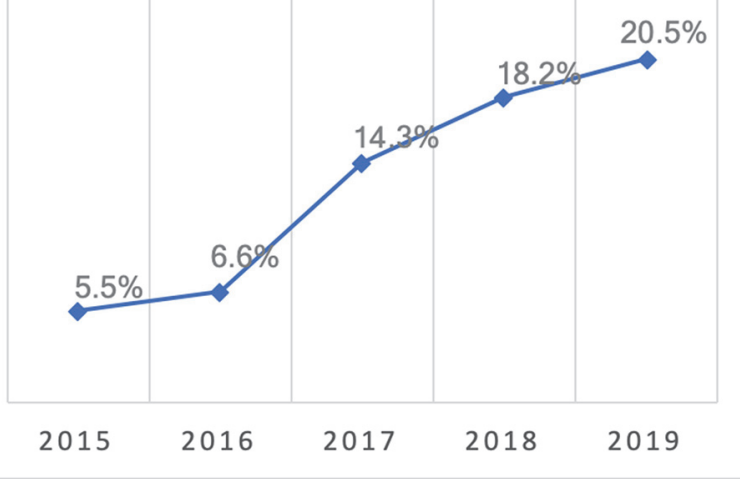

Abstract 26 Figure 1 Temporal analysis
Conclusion There was a statistically significant increase in the use of SGLT2 inhibitors since the EMPA-Reg study. Recent published data on the benefits this drug class confers on HF management further strengthens the evidence for this change in practice. The new ESC guidelines were published in September 2019, which we suspect will result in even more widespread use of these drugs.

\section{\begin{tabular}{|l|l}
27 & WHAT IS THE RELATIONSHIP BETWEEN CHRONIC
\end{tabular} KIDNEY DISEASE AND SUBSEQUENT EVENTS IN A CORONARY HEART DISEASE POPULATION? A SECONDARY ANALYSIS OF THE EUROASPIRE V DATA}

${ }^{1,2} \mathrm{M}$ Coyle, ${ }^{2} \mathrm{C}$ Jennings, ${ }^{3} \mathrm{G}$ Avalos, ${ }^{4} \mathrm{JW}$ McEvoy. ${ }^{1}$ School of Medicine, National University of Ireland Galway, Ireland; ' National Institute for Prevention and Cardiovascular Health, Galway, Ireland; ${ }^{3}$ Department of Medicine, National University of Ireland, Galway Campus., Galway Ireland; " University of Ireland, Galway Campus, Galway, Ireland, ESC National Coordinator for CVD Prevention, Ireland

\subsection{6/heartjpl-2020-ICS.27}

Introduction Chronic kidney disease (CKD) is an important risk factor for cardiovascular disease (CVD). The EUROASPIRE V study, conducted across 27 European countries (20162017), investigated whether the European guidelines on secondary CVD prevention were being met.

Aims The aim of this secondary analysis of the EUROASPIRE $\mathrm{V}$ data was to compare the CVD event-rate in those with and without CKD (defined as an eGFR $<60 \mathrm{ml} / \mathrm{min} / 1.73 \mathrm{~m}^{2}$ ).

Methods A cohort study was conducted of patients who were interviewed between 6-24 months after an index

Abstract 27 Table 1 Prevalence of cardiovascular risk factors in the participants

\begin{tabular}{lll}
\hline Risk factor & Number (n) & Percentage (\%) \\
\hline Family history of premature CVD* & 3223 & 44.3 \\
Smoking history & 5416 & 65.6 \\
Current smokers & 1385 & 25.9 \\
History of diabetes & 2661 & 32.3 \\
History of being overweight/obese & 7074 & 85.7 \\
Low SES* & 3339 & 40.5 \\
High psychosocial risk & 1309 & 15.9 \\
History of hypertension & 7124 & 86.3 \\
History of dyslipidaemia & 7308 & 88.6 \\
Ongoing systolic hypertension & 2775 & 35.1 \\
Ongoing physical inactivity & 5814 & 70.5 \\
\hline
\end{tabular}

${ }^{*} \mathrm{CVD}$, cardiovascular disease. ${ }^{* *} \mathrm{SES}$, socioeconomic status.

\begin{tabular}{|c|c|c|c|c|}
\hline $\mathrm{CV}^{*}$ death mean eGFR ${ }^{* *}(\mathrm{SD})$ & No CV death mean eGFR (SD) & Mean difference & $95 \% \mathrm{Cl}$ & $P$ value \\
\hline $65.66(27.53)$ & $80.55(22.87)$ & 14.89 & $(6.50,23.37)$ & 0.001 \\
\hline Stroke mean eGFR (SD) & No stroke mean eGFR (SD) & Mean difference & $95 \% \mathrm{Cl}$ & $P$ value \\
\hline $73.27(22.37)$ & $80.50(22.88)$ & 7.23 & $(2.16,12.31)$ & 0.006 \\
\hline $\mathrm{MI}^{* * *}$ mean eGFR $(\mathrm{SD})$ & No MI mean eGFR (SD) & Mean difference & $95 \% \mathrm{Cl}$ & $P$ value \\
\hline $78.88(26.15)$ & $80.44(22.90)$ & 1.56 & $(-3.03,6.14)$ & 0.503 \\
\hline
\end{tabular}

${ }^{*} \mathrm{CV}$, cardiovascular. ${ }^{* *}$ estimated glomerular filtration rate. ${ }^{* * *} \mathrm{MI}$, myocardial infarction. Independent samples t-test was used to compare eGFRs in those who recurrent events with those that did not $(p<0.05)$. 